\title{
Construction of an universal blood collecting system with integrated analytics
}

\author{
Susanne Schlegel ${ }^{1}$, Eric Krumnow ${ }^{1}$, Steffen Zinn ${ }^{1}$, Markus Riester $^{2}$, Marilena \\ Minieri $^{3}$ and Andreas H. Foitzik ${ }^{1}$
}

\author{
1 Technical University of Applied Science Wildau (THWi) - Hochschulring 115745 Wildau, Germany \\ 2 midge medical GmbH, Bessemerstrasse 22, 12103 Berlin, Germany \\ 3 Università degli Studi di Roma "Tor Vergata" (URTV) - Via Orazio Raimondo 18 - 00173 Roma, Italy
}

\begin{abstract}
This paper presents the construction of a new medical application system (MAS), unifying blood collecting, sample preparation and analytics in one device. Additionally, the implemented analytical device should be a commercially available lateral flow assay (LFA). Therefore, a universal case has to be designed. A needle system should extract the blood from the patient, while capillary forces transfer the sample through internal feeding into a reservoir. Afterwards, a buffer flushes the reservoir and carries the analyte through the migration distance to the test row. The developed setup utilizes microfluidic mixing structures as for further sample preparation. Thereby, the risk of contamination for the patient and medical personnel could be significantly reduced. Consequently, trained medical personal should not be necessary to operate this setup and increase the overall filed of application. This is especially fundamental when considering an application in case of epidemic events whereas a reliable medical treatment is often not reliable.
\end{abstract}

Keywords: blood collection, sampling, analysis, LFA, medical device

\section{Introduction}

Due to the proceeding of globalization, from time to time the epidemic rise of diseases occurs all over the world. Tropical diseases, for example, are not anymore just a problem of the country of origin [1]. Since more than half of the world population still do not have access to essential medical care [2], the risk of a pandemic outbreak increases even further. In order to prevent such a disastrous event, it is required to identify spreading diseases in a fast and reliable manner. Usually, the sample preparation for biological and chemical probes involves following a strict workflow to eliminate any contamination beforehand $[3,4]$. Furthermore, it must be carried out by trained personnel such as a nurse or other health care personnel, which is expensive as well as time consuming. Therefore, a new medical application system (MAS) is necessary to provide medical care and clarification in terms of a pandemic outbreak.

\section{Actual situation}

For the analysis of the medical conditions saliva and blood samples can be used [3], which should be regarded as samples of high complexity. Well-equipped laboratories could for example prepare hemograms to provide an overview about the general medical status of the patient. [4] However, more than half of the world population has no access to essential medical care [2], consequently those complex medical analytics are not available in many regions, especially in developing countries. Hence, simple analytical methods are needed.

A simple method to perform blood analysis is the lateral flow assay (LFA) test. The LFA test has the advantage of low costs as well as a shorter execution period compared to other methods. Furthermore, the technique doesn't require other additional sophisticated equipment [5]. However, the blood sampling and its analysis should only be performed by medical trained personal, since the blood sampling must follow an exact operating procedure. Not carrying out the procedure correctly could result in an infection of the patient or contamination of the sample itself. Furthermore, a non-adherence of the operating procedure could influence the result of the test. False negative or false positive results lead to a faulty rehabilitation of the patient. Therefore, the procedure of the LFA test should generally be performed by medical trained personal. 
Although the LFA test is simpler and more cost-efficient compared to other methods, it still requires medical resources, execution time and funding. This may be problematic in case of an upcoming epidemic. As medical care is not comprehensively assured, the necessary medical resources should be transferred to the affected regions. For that reason, medical personal and tests are sent out with high expense. It should also be considered, that the need of medical trained persons, equipment, financial funding and execution time rise with the increasing patient count. [5]

\section{Requirements for the medical analytical system}

The lack of the useful medical analytic systems is setting the requirements of prospective MAS. An improved usability provides a better reliability as well as improved overall performance. For this reason, financial effort could be reduced since medical trained personal is not necessary any more. Thereby, with the help of simpler and faster MAS a high number of patients could be tested in a short period of time and identified an epidemic in an early stage. Therefore, the MAS should also contain a form of identification system in order to provide a clear apportionment of the obtained data. Figure 1 displays schematically the concept of such a MAS.

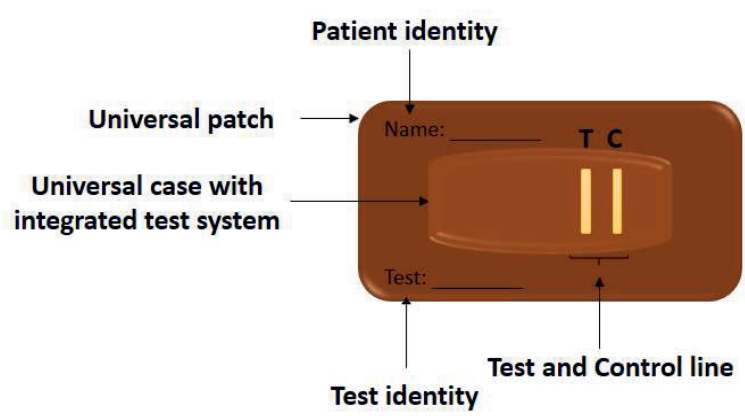

Figure 1: Schematically patch structure of a prospective MAS (top view)

Generally, the MAS should be small in size and cost-efficient. Furthermore, the operation procedure of the MAS should be simple and intuitive. Another important requirement of the MAS is the functionality in case of extreme climatic conditions, so that it can be utilized in a variety of climatic regions. However, the most important requirement is the combination of the blood sampling procedure and the subsequent examination in one system. Therefore, the blood must be transferred to the test strip directly after the extraction. Figure 2 schematic displays the functional unit of such a system with an integrated LFA as well as an extraction via needle.

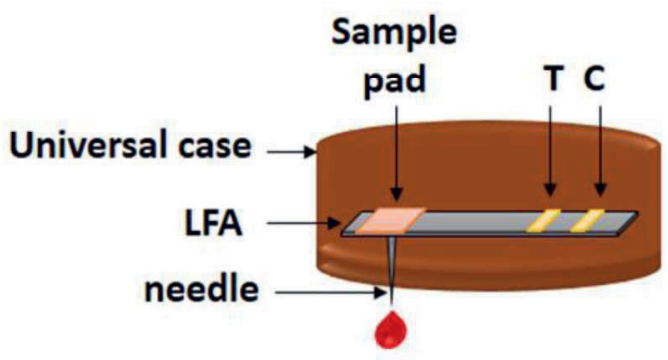

Figure 2: Schematically functional unit of a prospective MAS (top view)

The procedure of blood sampling should be automated and minimal invasive, whereby scars on the skin can be reduced and the risk of successive infections on sampling point will be minimized. The blood sampling procedure must be designed simplistic as well as as single-use in order to prevent transmission of infections between several patients. Therefore, a medical trained person is not required to perform the sample extraction. A temperature, dust and humidity resistant case protects the test from detrimental environmental effects in order to provide the function of the assay under different climatic conditions.

The intended functionality of the MAS assures reliable results because of the fully automated operating procedure, which further minimize potential errors. Such a system could be arranged in size and structure as an adhesive tape. [6]

\section{Realization strategy}

The overall development strategy is separated into four phases. Hereby, the first phase of development is focused on the conception and realisation of the housing. In general, the housing of the MAS is determined by the size of the integrated functional components, the material properties and requirements for the internal mounts. Furthermore, the housing should be sealed in order to protect the sensitive interior from detrimental environment (dust, humidity, etc.). Regarding the design of the superficies of the MAS a good handling and space-saving geometry should be considered, which resulted in two general geometries.

The first approach is based on a circularly cylindrical geometry and provides the advantage of an overall good handling but a rather limited installation space. In comparison, the second concept utilizes a rectangular cylindrical geometry. However, considering the 
shape of the LFA test stripes as well as possible mounting strategies the first approach has been selected as the first designs of the housing. A first concept is schematically shown in figure.

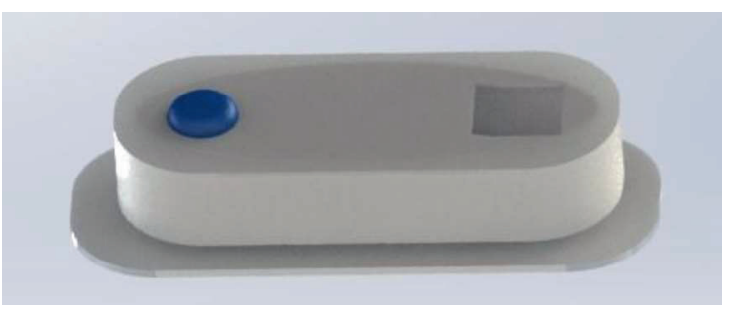

Figure 3: Side view of possible housing design from the prospective MAS

The base plate with the adhesive tab $s$ located at the bottom to position the MAS directly on the skin of the patient. Furthermore, it exceeds the limits of the rest of the whole device in order to increase the contact area. Therefore, the MAS could be positioned in a stable position directly on the skin of the patient. During the blood sampling a fixed position is essential to minimize the lesion of the skin. A shift of the blood sampling system during the penetration of the skin could otherwise cause an increased lesion and therefor enhances the risk of an infection.

Another element of the housing is an integrated window, though which an optical readout of the LFA strip inside the MAS is provided.

Additionally, a mechanical switch is integrated into the top of the MAS to initiate the sample extraction which can be either carried out via a needle or a lancet. The realisation of the blood sampling system is part of the second development phase. It contains the extraction device, a mechanical release mechanism as well as a safety-mechanism.

In regard to the aspired field of application it is intended to extract the blood samples directly via the patient skin [6]. The skin is the largest organ in the human body and consist of three layers, the epidermis, the dermis and the subcutaneous layer. Furthermore, the thickness of the skin depends on the actual skin type and the specific location on the body [7]. Considering the intended application, the extraction of a blood sample should be reproducible and not impaired by varying thickness, which would otherwise affect the subsequent analysis [8]. Additionally, the lesion caused by the penetration of the skin should be as small as possible to decrease the risk of an infection. The challenge is to adjust these two demands of the blood sampling system. Figure 4 displays the intended process chain for the MAS.

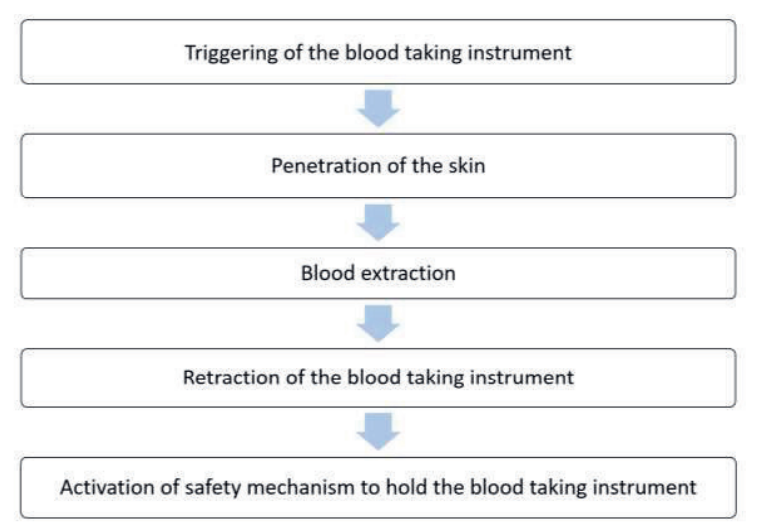

\section{Figure 4: Process flow diagram of the blood sampling procedure}

The first step is the activation of the extraction procedure by pressing the mechanical switch on top of the MAS. This motion is directed onto a mechanical transmitter in order to limit the movement of the extraction element. Otherwise, a reliable penetration of the skin cannot be guaranteed which could cause lesions or an insufficient extraction. In the next step, the extraction element is pressed downwards and penetrates the skin. This position is hold for certain period of time until enough blood has been extracted. Afterwards, the extraction element is retraced and secured inside the housing in order to prevent a second skin penetration or further usage of the MAS. Therefore, the expulsion mechanism contains a mechanical fuse which will block the overall movement of the extraction element after the retraction.

For the penetration needles or lancets could be used [8]. Future subsequent experiments will show which kind of blood taking instrument fulfils the functional requirements. For further consideration a needle as extraction element has been be taken.

The third development phase is focused on the transport of the sample to the integrated analytical component. Currently, it is intended to utilize a microfluidic cartridge which contains multiple chambers as well as passive valves and venous valves to retain a backflow of the sample. An exemplary structure is shown in figure 6 . In this approach, capillary forces are utilized to transfer the samples though the channels and accumulate the extracted volume in the first so called "waste" chamber. If this first chamber is completely filled, the blood continuous to flow into the actual "sample chamber". Using this approach, it is intended to provide a defined sample volume for further analysis, otherwise an inadequate amount of blood could inaccurate the outcome. As last 
step of the process, buffer will flush the sample chamber and transport the samples to the analytical system.

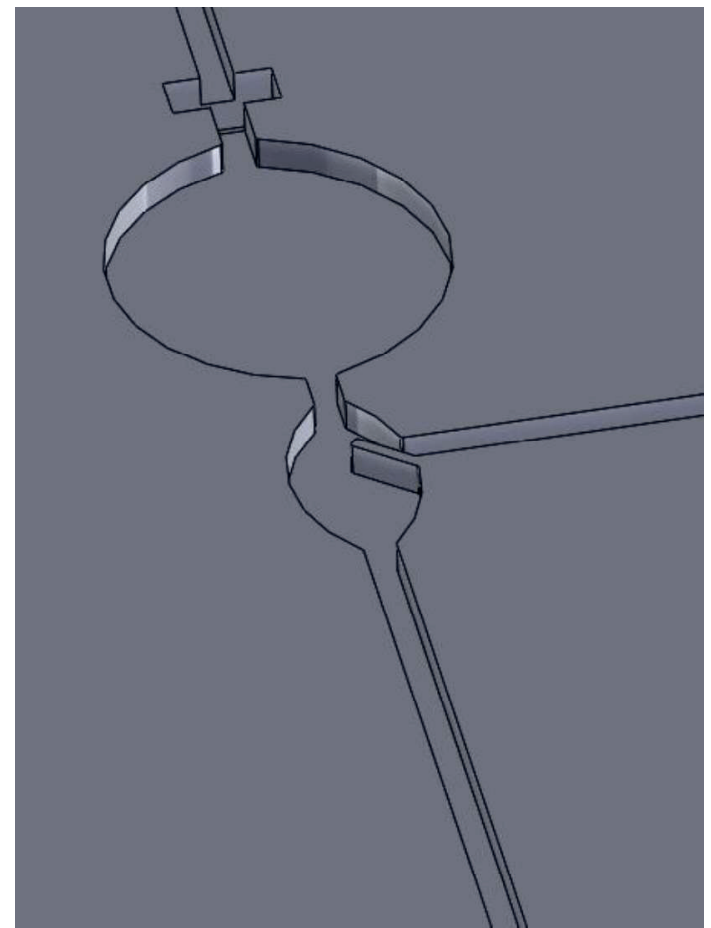

Figure 5: Microfluidic structure form the sample chamber with passive and venous valves

The last element of the development strategy contains the analytical setup. This is currently based on a commercial sandwich LFA test. The general functionality of such a sandwich LFA test is shown in Fig.6. In case of a successful analysis usually two separate lines are visible (the test and the control line). If the blood sample is negative the test only shows the so called control line. LFA tests are usually regarded invalid when no line or only the test line is visible. [5]

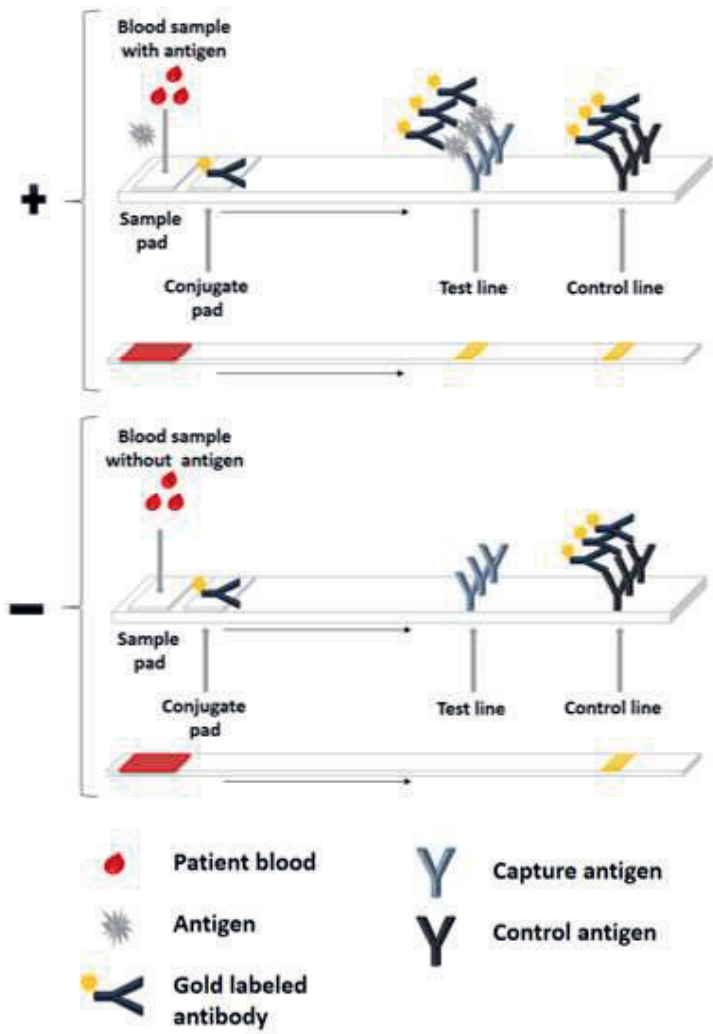

Figure 6: Schematic representation of a sandwich lateral flow assay (LFA). The first LFA shows two lines (the test and the control line) and is positive (+). The second test shows only one line (the control line) and it is negative (-).

The concept for a mount as well as connection with the sample transport will be constructed with computational aided design (CAD). Afterwards, the sample transfer from the extraction point to the analytical assay will be simulated in order to identify technical difficulties and to optimize the overall flow conditions.

The designed MAS is currently realized utilizing 3D-printing technologies. However, after a successfully verification of the fitting accuracy the individual components will be manufactured out via injection molding. Subsequent performance experiments are afterwards also intended.

\section{Summary}

A combination of preparation techniques and analysis systems could improve the overall process of blood sampling. This is especially important when considering the situation of sample analysis in case of pandemic prediction 
or other catastrophic events. However, the preparation for such sensitive sample requires a strict workflow in order to eliminate the risk of contamination. By utilizing microfluidic sample and preparation systems the risk of contamination for the patient and medical personnel could be reduced significantly. Furthermore, MAS are less time consuming and don't require medical trained personnel.

A theoretical approach utilizing a LFA test as an analytical element has been drafted. The designed microfluidic device is currently manufactured utilizing 3D-printing and will be later on carried out via injection molding. Subsequent experiments are also intended in order to verify the overall performance of the realized designs.

\section{References}

[1] Diesfeld, H.J., Krause, G., Teichmann, D.: Praktische Tropen- und Reisemedizin. Thieme, Stuttgart 2003

[2] Information on:

http://www.who.int/mediacentre/factsheets/fs39 5/en/, actualization on Dec. 2017 from WHO, date of access: 12.01.2018

[3] J. Pawliszyn, "Sampling and sample preparation for field and labatory: fundamental and new directions in sample preparation," in hardbound, 2nd ed. vol. 37, J. Peters, Ed. D. Barceló, Amsterdam: Elsevier Science B.V. 2002, pp. 799-825.

[4] L. Thomas, Labor und Diagnose, Th-Books, 6. Auflage, 2005

[5] Development and application of lateral flow test strip technology for detection of infectious agents and chemical contaminants: a review, Babacar Ngom, Yancheng Guo, Xiliang Wang, Dingren Bi, June 2010, Volume 397, Issue 3, pp 1113-1135

[6] Personalization of phlebotomy using smart systems integration, Dr. Markus Riester, meisterwerk ventures $\mathrm{GmbH}$ (DE), Jörn Bungartz, midge medical GmbH Berlin (DE), Preprint of Smart Systems Integration Conference, Cork, Ireland, 8 - 9 March 2017

[7] Shier, D., Butler, J. \& Lewis, R. in Hole's Human Anatomy and Physiology 8th Edn 171-183 (McGraw Hill, 1999)

[8] WHO guidelines on drawing blood: best practices in phlebotomy. Switzerland 2010. Bookshelf ID: NBK138661 